This item was submitted to Loughborough's Research Repository by the author.

Items in Figshare are protected by copyright, with all rights reserved, unless otherwise indicated.

\title{
Salivary immunoglobulin A and upper respiratory symptoms during 5 months of training in elite tetraplegic athletes
}

PLEASE CITE THE PUBLISHED VERSION

http://journals.humankinetics.com/ijspp-back-issues/ijspp-volume-7-issue-3-september/salivary-

immunoglobulin-a-and-upper-respiratory-symptoms-during-5-months-of-training-in-elite-tetraplegic-athletes

\section{PUBLISHER}

(c) Human Kinetics, Inc.

VERSION

VoR (Version of Record)

\section{LICENCE}

CC BY-NC-ND 4.0

\section{REPOSITORY RECORD}

Leicht, Christof A., Nicolette Bishop, Thomas A.W. Paulson, Katharine E. Griggs, and Victoria L. GooseyTolfrey. 2019. "Salivary Immunoglobulin A and Upper Respiratory Symptoms During 5 Months of Training in Elite Tetraplegic Athletes". figshare. https://hdl.handle.net/2134/10943. 
This item was submitted to Loughborough's Institutional Repository (https://dspace.lboro.ac.uk/) by the author and is made available under the following Creative Commons Licence conditions.

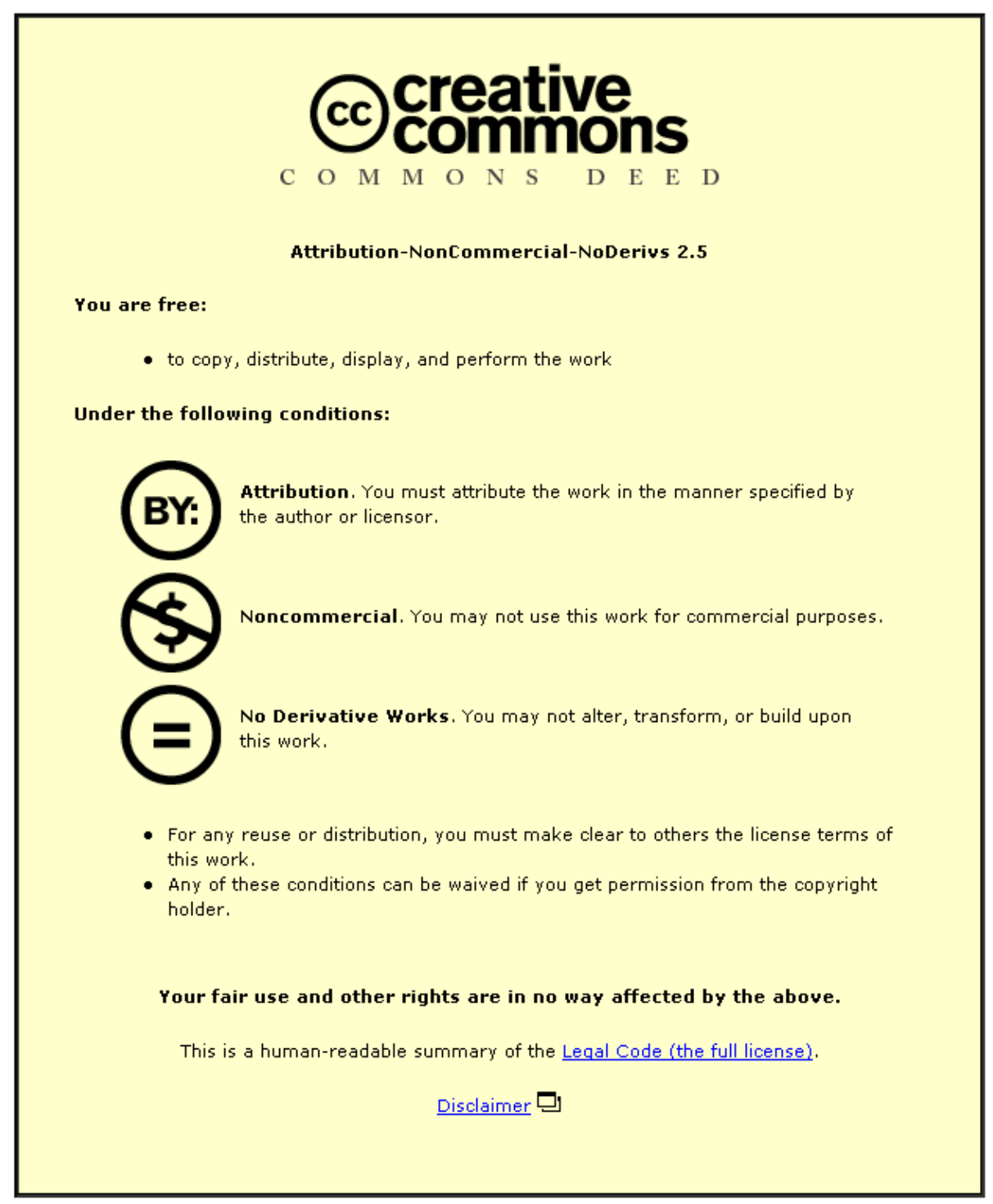

For the full text of this licence, please go to: http://creativecommons.org/licenses/by-nc-nd/2.5/ 


\title{
Salivary Immunoglobulin A and Upper Respiratory Symptoms During 5 Months of Training in Elite Tetraplegic Athletes
}

\author{
Christof A. Leicht, Nicolette C. Bishop, Thomas A.W. Paulson, \\ Katy E. Griggs, and Victoria L. Goosey-Tolfrey
}

\begin{abstract}
Purpose: Altered autonomic innervation in tetraplegic individuals has been shown to depress certain immune parameters at rest and alter exercise-related salivary immunoglobulin A ( $\operatorname{sgA}$ ) responses. The purpose of this study was to examine resting SIgA responses as a function of training load and episodes of upper respiratory symptoms (URS) in elite tetraplegic athletes. Methods: Resting saliva samples were obtained from 14 tetraplegic athletes at 12 predefined time points over 5 months and analyzed for sIgA. Occurrence of self-reported URS and training load was recorded throughout the study's duration. Regression analyses were performed to investigate the relationship between $\operatorname{sIgA}$ responses and training load. Furthermore, the relationships between sIgA responses and URS occurrence were examined. Results: SIgA secretion rate was negatively correlated with training load $(P=.04)$, which only accounted for $8 \%$ of the variance. No significant relationships were found between sIgA responses and subsequent URS occurrence. Finally, sIgA responses did not differ between athletes with and without recorded URS during the study period. Conclusions: In line with findings in ablebodied athletes, negative relationships between SIgA secretion rate and training load were found in tetraplegic athletes. This may explain some of the higher infection risk in wheelchair athletes with a high training load, which has been previously observed in paraplegic athletes. However, the nonsignificant relationship between sIgA responses and URS occurrence brings into question the use of $\operatorname{sgA}$ as a prognostic tool for the early detection of URS episodes in the studied population.
\end{abstract}

Keywords: exercise, mucosal immune function, stress, fatigue, wheelchair rugby

Salivary secretory immunoglobulin A $(\operatorname{sIg} \mathrm{A})$ is the predominant immunoglobulin in saliva and other mucosal secretions. It plays an important role in mucosal immunity and has therefore been described as a first line of defense against pathogens and antigens presented at the mucosa, such as cold-causing viruses. ${ }^{1,2}$ While a number of studies have found no relationships between upper respiratory symptoms (URS) and $\operatorname{sgA},{ }^{3,4}$ longer-lasting longitudinal studies have been able to associate preceding decreased resting levels of sIgA with URS. ${ }^{5,6}$ Consequently, $\operatorname{sIgA}$ has been suggested to be a clinical biomarker to predict incidence of URS. ${ }^{5}$ This is of practical relevance, as URS are among the most common medical problems in elite able-bodied athletes. ${ }^{7}$ URS may lead to missed training sessions and compromises in athletic performance. Furthermore, according to some surveys, sore throats and flulike symptoms are more common in athletes than in the general population, and, once athletes are infected, their colds may last longer. ${ }^{8}$

The authors are with the School of Sport, Exercise, and Health Sciences, Loughborough University, Loughborough, UK.
Positive relationships between URS incidence and high training loads have been found in athletic ablebodied $^{5}$ and paraplegic ${ }^{9}$ populations. Furthermore, negative relationships between training load and $\operatorname{SIgA}$ concentration or training load and $\operatorname{sIgA}$ secretion rate have been documented previously. ${ }^{5,6}$ In this context, it is important to know that during periods of heavy training, the sympathovagal balance at rest seems to be altered, with a rise in sympathetic and suppression of parasympathetic drive..$^{10}$ Both parasympathetic and sympathetic nerve stimulation can modify saliva composition and sIgA secretion. ${ }^{11}$ This may lead to the conclusion that high training loads, altered sympathovagal balance, depressions in sIgA concentration or SIgA secretion rate, and a higher incidence of URS are causally connected.

Since the sympathetic outflow in tetraplegic individuals is decreased, ${ }^{12}$ any stress-related sIgA response at rest has the potential to be altered in comparison with populations with intact autonomic innervation. However, this issue has not been investigated until present. This would be of particular relevance, as spinal-cordinjured individuals are prone to respiratory diseases such as dyspnea, pneumonia, or respiratory failure. ${ }^{13}$ 
In a sporting context, it should be noted that in line with findings in able-bodied athletes, ${ }^{7}$ anecdotal evidence from the national governing sports bodies' training-log records shows a high proportion of URS-related dropouts in athletic tetraplegic populations during heavy training periods and competition.

Knowledge of oral-respiratory mucosal immune responses to exercise in tetraplegic athletes could serve as a base for health promotion and monitoring in this specific population. Indeed, we recently found a compensatory effect on $\operatorname{sigA}$ responses to acute exercise in elite tetraplegic athletes. ${ }^{14}$ Therefore, the purpose of this study was to examine resting $\mathrm{SIgA}$ responses as a function of training load and episodes of URS during a 5-month training period in tetraplegic athletes.

\section{Methods}

\section{Participants}

Fourteen tetraplegic wheelchair rugby players (referred to as participants $\mathrm{A}-\mathrm{N}$ throughout the article) volunteered to participate in this study, which was approved by the university's ethics committee. The group consisted of 13 men and 1 woman (participant I) who were all members of the Great Britain wheelchair rugby squad. A summary of their physical, physiological, and sport characteristics is presented in Table 1.

\section{Data Collection}

Data were collected at 12 predefined time points over 5 months during the squad's training building up to the World Championships in September 2010 (Figure 1). Timed, unstimulated saliva samples were collected between 2 and 5 minutes (depending on the individuals' saliva flow rate) into sterile plastic containers. For this, participants rinsed their mouth with water and sat still with their head slightly tilted forward with minimal orofacial movement. All samples were collected before

Table 1 Participant Characteristics

\begin{tabular}{lc}
\hline Parameter & Value \\
\hline Body mass, kg & $70.4 \pm 13.2$ \\
Height, $\mathrm{m}$ & $1.81 \pm 0.12$ \\
Age, $\mathrm{y}$ & $33 \pm 5$ \\
Lesion level & $\mathrm{C} 6-\mathrm{C} 7$ \\
Motor completeness of injury & \\
$\quad$ yes & $\mathrm{n}=13$ \\
$\quad$ no & $\mathrm{n}=1$ \\
Time since onset of disability, y & $12 \pm 5$ \\
Wheelchair rugby classification & $0.5-2.5$ \\
Training, h/wk & $14 \pm 3$ \\
\hline
\end{tabular}

Apart from lesion level and wheelchair rugby classification (range), numbers are average \pm SD.

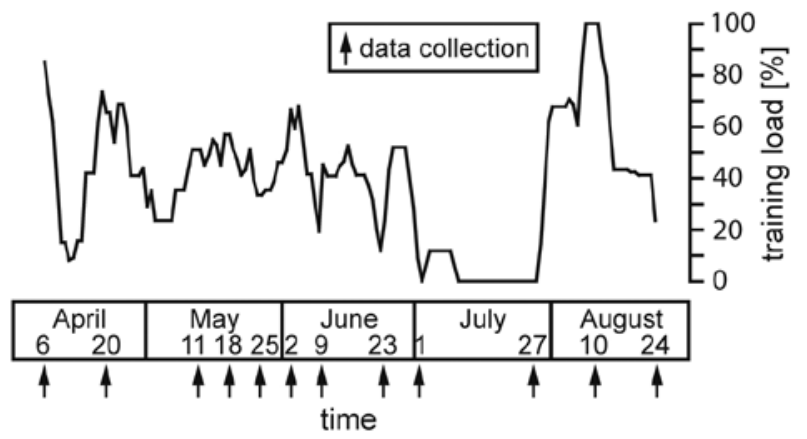

Figure 1 — Study design. Training load is given for an example participant.

11 AM in a fasted state and before any physical activity. Samples were stored on ice immediately after collection and stored at $-20^{\circ} \mathrm{C}$ within 6 hours.

On each data-collection day, participants filled out exercise questionnaires in retrospect; a sample questionnaire was provided for guidance. For each day individually, average intensity for any given exercise (such as individual workouts or game play) was rated on a 5-point scale $(1=$ very low, $2=$ low, $3=$ medium, $4=$ hard, 5 = very hard), and exercise duration was noted. Training load was then determined by multiplying intensity by duration, ${ }^{15}$ and a rolling average over 7 days was computed for the entire duration of the study (Figure 1). The $100 \%$ training load for each individual was defined as the highest 7-day average attained during the 5-month study period. Finally, the location of the athletes and any sport-related international travels were recorded.

Illness questionnaires were also filled out in retrospect. The criteria for definition of an episode of URS have been published elsewhere. ${ }^{16}$ In short, the selfreported occurrence of symptoms like sore or scratchy throat, runny/plugged nose, cough, or fever was noted and their intensity was rated on a 3 -point scale ( 1 = light, 2 $=$ moderate, $3=$ severe). Multiplying the intensity score by the number of symptoms and the number of days suffered, a minimum score of 12 was taken to indicate that a URS was present. Finally, according to the methods employed by Neville et al, ${ }^{6}$ participants used a 3-point scale to indicate their resting status on the day of data collection (worse than, same as, or better than normal).

With the exception of 4 dates (April 20, May 11, June 23, and August 10), data were collected on scheduled training days at the training venue. When participants were unable to attend these sessions, as well as on the dates mentioned before, saliva samples were collected individually by the participants. Detailed written instructions were provided, and we ensured that procedures for correct sample storing were in place.

\section{Analytical Methods}

For analysis, samples were defrosted and weighed to the nearest $10 \mathrm{mg}$. Saliva volume was estimated assuming 
saliva density to be $1.00 \mathrm{~g} / \mathrm{mL},{ }^{17}$ and saliva flow rate was calculated from saliva volume and collection time. Samples were then spun for 2 minutes at $13,400 \mathrm{rpm}$. sIgA concentration was determined through a sandwich ELISA, using flat-bottomed microtitration plates (NuncImmunoplate, Thermo Fisher Scientific, Denmark). After the plates were coated with rabbit anti-human-capture antibody (Dako UK, Ely, UK), they were washed and blocked with a blocking protein solution $(2.0 \%$ bovine serum albumin in phosphate-buffered saline). Depending on saliva flow rate, saliva samples were diluted in phosphate-buffered saline by 1:375 to 1:1500 (samples with a lower flow rate were diluted by a higher factor). Purified IgA from colostrum was used as a standard (Sigma-Aldrich, St Louis, MO). Duplicate samples of $50 \mu \mathrm{L}$ were applied to the plates, which were incubated overnight at $4^{\circ} \mathrm{C}$. After the plates were washed, detection antibody (Dako UK, Ely, UK) was applied and plates were incubated at $25^{\circ} \mathrm{C}$ for 90 minutes. After a final wash, a coloring substrate (OPD substrate, Dako UK) was added, and the absorbance of the individual samples was determined spectrophotometrically at 490 nm (Opsys MR, Dynex Technologies Inc, Chantilly, VA). sIgA secretion rate was calculated by multiplying SIgA concentration by saliva flow rate.

All saliva samples from each participant were analyzed in duplicate on 1 microplate. The coefficient of variation $(\mathrm{CV})$ of this method based on analyses of these duplicate samples was $2.8 \% \pm 2.7 \%$.

\section{Data Processing and Statistical Analyses}

The PASW 18.0 statistical package (SPSS Inc, Chicago IL) was used for all statistical analyses. Means and standard deviations were computed for normally distributed variables, and medians and quartiles for all other variables. Furthermore, CVs were calculated. For each sample, salivary data (sIgA secretion rate and concentration) were then matched to the individual average 7-day training load before sample collection. A logarithmic transformation was applied to these salivary data to weigh increases by a certain factor the same as decreases by the same factor. Slopes of the linear regression of the log-transformed salivary data versus individual training load were calculated for each participant individually, and the slopes were tested with a Wilcoxon statistic against a fixed value of zero; a Bonferroni correction was applied for multiple comparisons. The same procedure was applied to the relationships between saliva flow rate versus $\operatorname{sIg} \mathrm{A}$ concentration and saliva flow rate versus SIgA secretion rate.

Furthermore, median salivary data were compared between participants contracting at least 1 URS and participants not contracting at least 1 URS during the study period, using the Mann-Whitney test. Finally, all salivary data were pooled, expressed as a percent deviation from the individual median, and analyzed with Mann-Whitney tests to explore the impact of URS occurrence within
2 weeks after sample collection on salivary data. The same procedures were applied to investigate the impact of resting status on salivary data; however, due to the low occurrence of samples with a rating of better than normal $(n=3)$, these samples were excluded from this analysis. Statistical significance for all analyses was accepted at $P<.05$.

\section{Results}

Over the 5-month study period, 127 saliva samples were collected. The within-individual variability of sIgA secretion rate was high, with a mean $\mathrm{CV}$ of $54 \%$; the $\mathrm{CV}$ for between-individuals variability was $76 \%$ (Figure 2). The CVs for sIgA concentration were 55\% (within individual) and $88 \%$ (between individuals). Eighty percent of the sIgA secretion-rate data of participants D (incomplete spinal-lesion level) and I (female) were found to be within the 10th to 90th percentiles of the studied population, which was male with a complete spinal-lesion level. Therefore, their data were entered for all analyses.

A significant negative relationship was found between sIgA secretion rate and individual training load, with the average slope of the individual regression lines differing significantly from zero $(P=.04$, Figure 3$)$. However, on average, training load accounted for only $8 \%$ (quartiles: 4,18 ) of the variance. Furthermore, a trend was found between training load and SIgA concentration $(P=.06)$, again accounting for $8 \%$ (quartiles: 2,20$)$ of the variance.

A negative relationship was found between individual saliva flow rate and SIgA concentration, with the average slope of the individual regression lines differing significantly from zero $(P=.002$, Figure 4$)$. Furthermore, negative but nonsignificant relationships were detected when comparing average data of saliva flow rate and SIgA concentration between participants $(P=.43$, Figure 4). Finally, no significant relationship between individual saliva flow rate and $\operatorname{sIg}$ A secretion rate was found $(P$ $=.72$ ).

Three players reported at least 1 episode of URS during the study period (participants A, F, and K). However, their median sIgA secretion rate did not differ from that of the other players ( $P=.19$, Figure 2$)$. Furthermore, sIgA responses did not differ between samples with no subsequent episode of URS and samples with a subsequent episode of URS within 2 weeks $(P>.05)$. Likewise, no differences in SIgA responses were found when comparing samples of the different resting status ratings $(P$ $>.05$, Table 2). Finally, over the 5-month study period, players spent $20 \pm 12$ days abroad for competitions and used planes for transportation on $6.1 \pm 3.7$ occasions.

\section{Discussion}

The main finding of this study is that resting sIgA secretion rate is associated with training load in tetraplegic athletes, which is in line with findings in able-bodied 

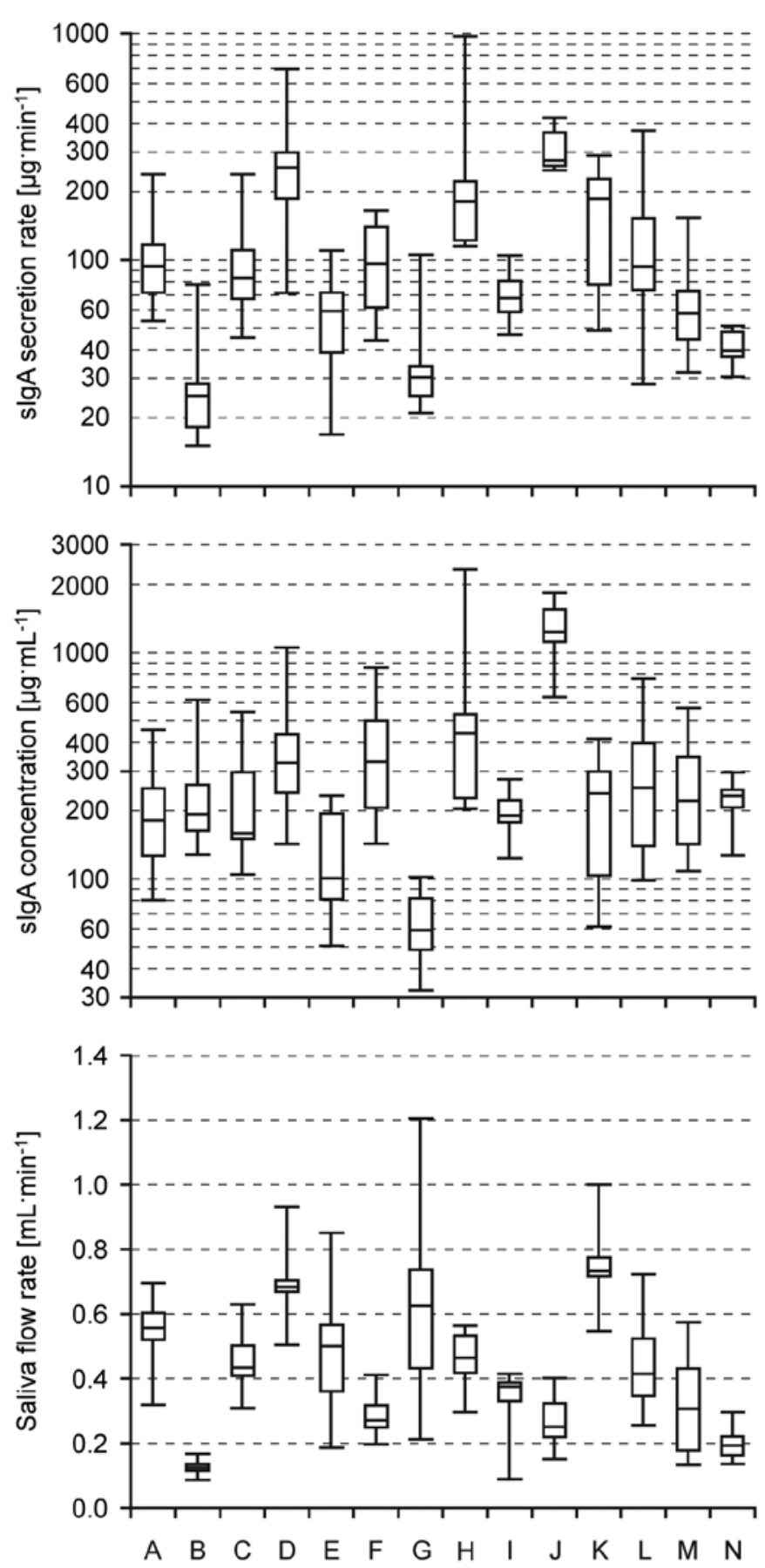

Figure 2 - Resting salivary immunoglobulin A (sIgA) secretion rate, sIgA concentration, and saliva flow rate over the 5-month study period. A-N are participant codes. Participants A, F, and K contracted at least 1 episode of upper respiratory symptoms during the study period. Values are displayed as box plots showing median, quartiles, and minimum/maximum.

athletes. ${ }^{4,6}$ However, we currently cannot confirm a relationship between decreased sIgA responses and the occurrence of subsequent URS. We further found saliva flow rate to explain some of the variation observed in sIgA concentration on an intraindividual basis.

It is commonly accepted that the sympathetic nervous system may at least partly be responsible for the changes in salivary markers such as $\operatorname{sig} \mathrm{A}^{2,18}$ and that both sympathetic and parasympathetic stimulation result in increased sIgA secretion. ${ }^{11}$ Tetraplegic individuals represent a model with no centrally mediated sympathetic nervous control, ${ }^{19}$ as centrally mediated sympathetic stimuli do not activate the decentralized part of the nervous system below the level of lesion. ${ }^{20}$ Furthermore, 


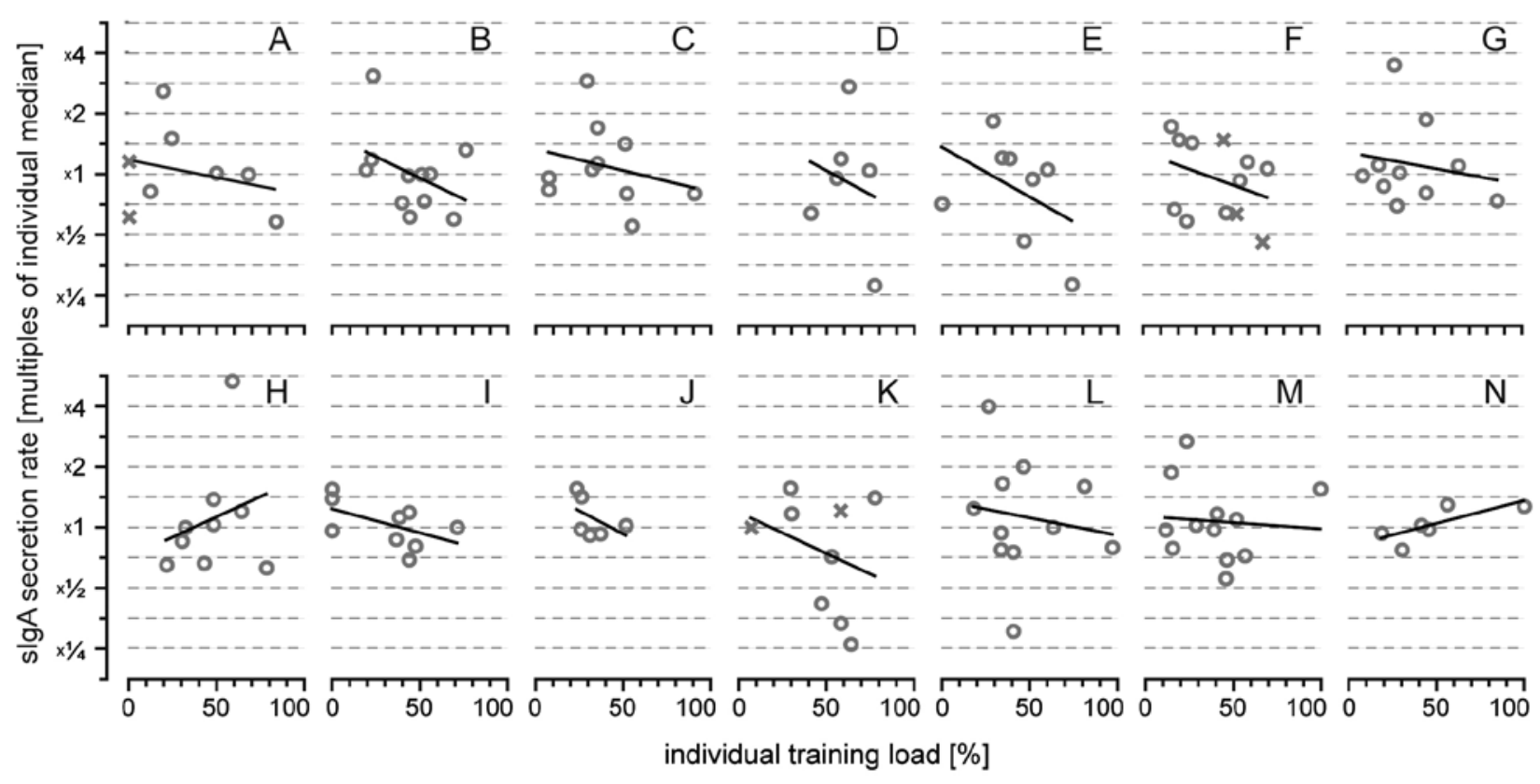

Figure 3 - Resting salivary immunoglobulin A ( $\operatorname{Ig} \mathrm{A})$ secretion rate versus individual training load over the 5-month study period, data points and individual regression lines. A-N are participant codes; circles, samples with no subsequent upper respiratory symptoms (URS); $\times$, samples with subsequent URS.

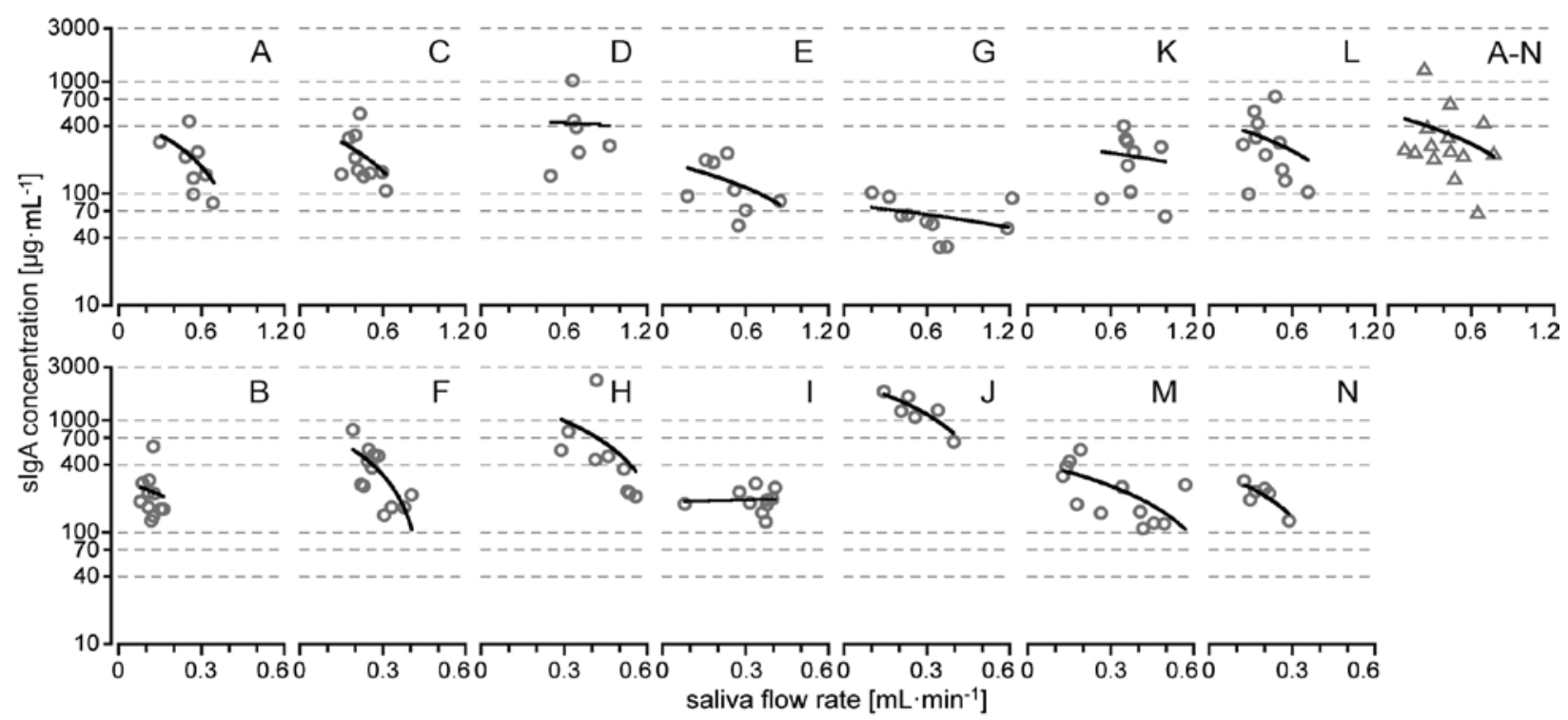

Figure 4 - Resting salivary immunoglobulin A (sIgA) concentration versus saliva flow rate over the 5-month study period, data points and linear regression lines. A-N are participant codes; circles, raw data; $\Delta$ (top right panel), average data shown for all individuals. Note the different scale of the $x$-axes in the top and bottom charts and the presentation of sIgA concentration on a logarithmic scale, which leads to a distortion of linear regression lines.

salivary gland innervation originates from the upper thoracic segments and is therefore disrupted in tetraplegic individuals. ${ }^{11}$ Therefore, it is important to note that with respect to salivary gland function, the studied population is distinctively different than the able-bodied populations studied earlier. However, it seems that these differences in tetraplegic athletes do not have a major impact on resting SIgA secretion rate and SIgA concentration when compared with the able-bodied population. Even though between- and within-subject variability of sIgA responses 
Table 2 Influence of Resting Status and the Occurrence of URS Within 2 Weeks on Salivary Data, Median (Quartiles)

\begin{tabular}{|c|c|c|c|}
\hline Resting status & $\begin{array}{c}\text { slgA secretion rate } \\
\text { (\% from individual median) }\end{array}$ & $\begin{array}{c}\text { slgA concentration } \\
\text { (\% from individual median) }\end{array}$ & $\begin{array}{c}\text { Saliva flow rate } \\
\text { (\% from individual median) }\end{array}$ \\
\hline Same as normal $(\mathrm{n}=92)$ & $101(80,128)$ & $100(71,149)$ & $100(85,118)$ \\
\hline Worse than normal $(n=30)$ & $92(72,120)$ & $98(74,121)$ & $100(91,120)$ \\
\hline Better than normal $(n=3)$ & excluded from analysis & excluded from analysis & excluded from analysis \\
\hline Missing data $(n=2)$ & excluded from analysis & excluded from analysis & excluded from analysis \\
\hline \multicolumn{4}{|l|}{ URS within 2 wk } \\
\hline No $(n=120)$ & $100(77,128)$ & $100(73,140)$ & $100(85,118)$ \\
\hline Yes $(n=7)$ & $100(62,118)$ & $100(64,125)$ & $106(94,112)$ \\
\hline
\end{tabular}

Abbreviations: URS, upper respiratory symptoms; sIgA, salivary secretory immunoglobulin A.

were high, they were in a range and of a variability similar to those of able-bodied athletes. ${ }^{6}$ We therefore suggest that the main modulation of resting $\operatorname{sigA}$ is likely to stem from variations in parasympathetic activity, as this remains normal in tetraplegic individuals ${ }^{19}$ and has been shown to be related to periods of heavy exercise. ${ }^{10}$ Another possible modulator is sympathetic reflex activity, which may be triggered by circulating metabolites related to stress or fatigue. ${ }^{21}$ Since this reflex activity is still intact in the tetraplegic population, it results in a remaining, but qualitatively altered, neural function..$^{21}$ This is supported by the suggestion that sympathetic reflex activity may be a reason for the qualitatively altered $\operatorname{sIgA}$ response after acute exercise in this population. ${ }^{14}$

It should be noted that the participants in this study were highly trained. The training loads we observed in this population were comparable to those observed previously in able-bodied participants in studies investigating sIgA responses to chronic exercise. ${ }^{3,4,6}$ In contrast, many studies on tetraplegic populations reporting depressed markers of immunity have been conducted in patient and/ or marginally trained populations. ${ }^{22,23}$ It must therefore be considered that long-term training in tetraplegic individuals may have a positive impact on these markers, through mechanisms like sympathetic reflex activity, ${ }^{21}$ which may be enhanced as a result of chronic exercise.

This study cannot confirm a cause-and-effect relationship between low sIgA responses and subsequent URS. However, the negative relationship between training load and sIgA secretion rate may explain some of the increase in URS after periods of hard training, as previously observed in both spinal-cord-injured ${ }^{9}$ and able-bodied athletes. ${ }^{24}$ Based on the impairments of the tetraplegic population, we conclude that the impact of exercise on $\operatorname{sIgA}$ secretion rate seems not to be influenced by sympathetic central drive, but we again suggest parasympathetic and sympathetic reflex activity as possible modulators. On the other hand, we do not rule out that underlying reasons for the lack of a relationship between $\operatorname{SIgA}$ responses and the occurrence of URS may include the altered sympathetic outflow in this specific population. However, it is important to note that even though relationships between sIgA responses and URS occurrence have been shown in able-bodied populations, ${ }^{6}$ this finding was not confirmed in other studies. ${ }^{3,4} \mathrm{We}$ therefore conclude that this relationship is not clear and potentially blurred by the additional stresses required for the occurrence of URS. It has been suggested earlier that the relationship between exercise and URS is affected by poorly known individual determinants such as genetic factors, fitness, and nutritional status. ${ }^{25}$ Factors concealing a clear relationship may further include pathogen exposure, environmental factors, or psychological strategies, ${ }^{26}$ which were not controlled for in the current study and therefore potentially differed between participants. We may also add that the studied athletes spent a considerable amount of time abroad due to their sport and therefore used planes for transportation rather frequently, which may represent additional stressors. ${ }^{27}$ As a final point, it should also be acknowledged that the presence of URS is not necessarily indicative of the presence of upper respiratory tract infection and may reflect airway inflammation rather than viral or bacterial infection. ${ }^{28}$ Nevertheless, regardless of cause, the deleterious effects of URS on training and competition are of main concern to athletes and coaches.

URS occurrence in the study period was comparably low, ${ }^{4,6}$ so another possibility is that insufficient statistical power did not reveal a significant relationship between increased URS incidence after occurrence of low sIgA responses. Sampling in winter may have resulted in more recorded URS. ${ }^{4,6}$ This again implies that a decreased sIgA response alone is not enough for subsequent episodes of URS. Additional stresses as stated previously ${ }^{26}$ may be necessary to obtain a higher occurrence of URS, which is more likely to happen during the winter months. ${ }^{6}$ It is also worth mentioning that there have still been very few studies that were able to show a direct link between exercise-induced immune depression and increased incidence of confirmed illness in athletes, which therefore needs addressing in future studies, although it must be recognized that this is difficult. ${ }^{8}$ 
The variation in saliva flow rate was pronounced in the studied population; notably, some very low saliva flow rates were recorded (eg, participants B, F, J, and N) compared with able-bodied athletes. ${ }^{4}$ We assume that this may be population related and due to altered innervation of the salivary glands, which also affects saliva flow rate. ${ }^{11}$ However, it appears that the production of sIgA is not affected to the same extent, because on both an intraindividual (negative significant relationship) and an interindividual (negative nonsignificant relationship) comparison, it is evident that sIgA concentration is higher in samples with a low saliva flow rate. In line with previous research in able-bodied populations,${ }^{2}$ it may therefore be that secretion rate is the more appropriate measure for sIgA than concentration in tetraplegic individuals, as it takes the pronounced alterations of saliva flow rate in this population into account.

\section{Practical Applications}

This study found depressions in sIgA secretion rate during periods of increased training load in elite tetraplegic athletes. Given the higher susceptibility for URS in this population, extra care should be taken during periods of heavy training to prevent illness. Countermeasures include training (ie, allow for adequate recovery) and environmental (ie, limit exposure to adverse conditions), psychological (ie, monitor stresses), behavioral (ie, reduce exposure to common infections), and clinical considerations (ie, conduct pathology testing). ${ }^{26}$ It is possible that some of these strategies have already been adopted by the participants on an individual basis, hence resulting in the low occurrence of URS in the current study. This may have been achieved by avoiding pathogen exposure, or by adopting strategies to prevent sIgA reaching levels low enough to increase the risk of contracting URS. However, to investigate the impacts of low sIgA responses on URS in the studied population, we encourage others to conduct further studies in the winter months to achieve a higher occurrence of URS, which would then improve the statistical power of the outcomes.

Self-reporting URS has been questioned earlier, ${ }^{8}$ and we are aware that because of self-reporting, we may have missed or misclassified some episodes of URS. A further source of error is the home collection of some of the saliva samples. By providing detailed instructions, we attempted to prepare our participants optimally to provide valid samples and illness reports. However, for future studies, we would encourage collaboration with trained medical staff for the definition of URS. Furthermore, even though we attempted to limit to a minimum the time saliva samples were stored on ice before they were frozen, we recommend attempting to reduce this time to avoid any potential inaccurate results stemming from storage-related sIgA breakdown. In addition, if feasible, hydration status should be assessed, as it may have an impact on oral-mucosal respiratory immunity. ${ }^{2}$
For future studies we further suggest analyzing more parameters relating to oral-respiratory mucosal immunity (ie, $\alpha$-amylase, lysozyme, lactoferrin), which may shed more light on the interaction of oral immune markers and URS. ${ }^{1}$ Markers of sympathetic nervous activity (ie, $\alpha$-amylase, chromogranin A) may provide further information about a mechanistic explanation of findings related to oral-respiratory mucosal immune function. Finally, the inclusion of a control group would strengthen the outcomes of any follow-up study.

\section{Conclusions}

Training load in tetraplegic wheelchair rugby athletes is negatively associated with sIgA secretion rate, as previously observed in able-bodied athletic populations. Furthermore, the range and variation of $\operatorname{sIgA}$ secretion rate and concentration are comparable to those in the existing able-bodied literature. We therefore conclude that a trained tetraplegic population's chronic oral-mucosal immunological response to exercise is comparable to that of able-bodied athletic populations. However, the nonsignificant relationship between sIgA and URS occurrence brings into question the use of sIgA as a prognostic tool for the early detection of upcoming URS in the studied population.

\section{Acknowledgments}

We thank the Great Britain Wheelchair Rugby Association for their collaboration and the Peter Harrison Centre for Disability Sport for financial support. Appreciation is extended to all athletes who volunteered to participate in this study.

\section{References}

1. Walsh NP, Gleeson M, Shephard RJ, et al. Position statement: part one: immune function and exercise. Exerc Immunol Rev. 2011;17:6-63. PubMed

2. Bishop NC, Gleeson M. Acute and chronic effects of exercise on markers of mucosal immunity. Front Biosci. 2009;14:4444-4456. PubMed doi:10.2741/3540

3. Gleeson M, McDonald WA, Pyne DB, et al. Immune status and respiratory illness for elite swimmers during a 12-week training cycle. Int J Sports Med. 2000;21:302-307. PubMed doi:10.1055/s-2000-313

4. Cunniffe B, Griffiths H, Proctor W, Davies B, Baker JS, Jones KP. Mucosal immunity and illness incidence in elite rugby union players across a season. Med Sci Sports Exerc. 2011;43:388-397. PubMed

5. Fahlman MM, Engels HJ. Mucosal IgA and URTI in American college football players: a year longitudinal study. Med Sci Sports Exerc. 2005;37:374-380. PubMed doi:10.1249/01.MSS.0000155432.67020.88

6. Neville V, Gleeson M, Folland JP. Salivary IgA as a risk factor for upper respiratory infections in elite professional athletes. Med Sci Sports Exerc. 2008;40:1228-1236. PubMed doi:10.1249/MSS.0b013e31816be9c3 
7. Robinson D, Milne C. Medicine at the 2000 Sydney Olympic Games: the New Zealand health team. Br J Sports Med. 2002;36:229. PubMed doi:10.1136/bjsm.36.3.229

8. Gleeson M. Immune function in sport and exercise. $J$ Appl Physiol. 2007;103:693-699. PubMed doi:10.1152/ japplphysiol.00008.2007

9. Furusawa K, Tajima F, Okawa H, Takahashi M, Ogata H. The incidence of post-race symptoms of upper respiratory tract infection in wheelchair marathon racers. Spinal Cord. 2007;45:513-517. PubMed doi:10.1038/sj.sc.3102028

10. Pichot V, Roche F, Gaspoz JM, et al. Relation between heart rate variability and training load in middle-distance runners. Med Sci Sports Exerc. 2000;32:1729-1736. PubMed doi:10.1097/00005768-200010000-00011

11. Proctor GB, Carpenter GH. Regulation of salivary gland function by autonomic nerves. Auton Neurosci. 2007;133:318. PubMed doi:10.1016/j.autneu.2006.10.006

12. Schmid A, Schmidt-Trucksass A, Huonker M, et al. Catecholamines response of high performance wheelchair athletes at rest and during exercise with autonomic dysreflexia. Int J Sports Med. 2001;22:2-7. PubMed doi:10.1055/s-2001-11330

13. Brown R, DiMarco AF, Hoit JD, Garshick E. Respiratory dysfunction and management in spinal cord injury. Respir Care. 2006;51:853-870. PubMed

14. Leicht CA, Bishop NC, Goosey-Tolfrey VL. Mucosal immune responses to treadmill exercise in elite wheelchair athletes. Med Sci Sports Exerc. 2011;43:1414-1421. PubMed doi:10.1249/MSS.0b013e31820ac959

15. Impellizzeri FM, Rampinini E, Coutts AJ, Sassi A, Marcora SM. Use of RPE-based training load in soccer. Med Sci Sports Exerc. 2004;36:1042-1047. PubMed doi:10.1249/01.MSS.0000128199.23901.2F

16. Gleeson M, Bishop NC, Oliver M, Tauler P. Daily probiotic's (Lactobacillus casei shirota) reduction of infection incidence in athletes. Int J Sport Nutr Exerc Metab. 2011;21:55-64. PubMed

17. Cole AS, Eastoe JE. Biochemistry and Oral Biology. 2nd ed. London: Wright; 1988.

18. Sari-Sarraf V, Reilly T, Doran DA, Atkinson G. The effects of single and repeated bouts of soccer-specific exercise on salivary IgA. Arch Oral Biol. 2007;52:526-532. PubMed doi:10.1016/j.archoralbio.2006.11.016

19. Krassioukov A. Autonomic function following cervical spinal cord injury. Respir Physiol Neurobiol. 2009;169:157-164. PubMed doi:10.1016/j. resp.2009.08.003

20. Corbett JL, Frankel HL, Harris PJ. Cardiovascular reflex responses to cutaneous and visceral stimuli in spinal man. J Physiol. 1971;215:395-409. PubMed

21. Karlsson AK, Friberg P, Lonnroth P, Sullivan L, Elam M. Regional sympathetic function in high spinal cord injury during mental stress and autonomic dysreflexia. Brain. 1998;121:1711-1719. PubMed doi:10.1093/ brain/121.9.1711

22. Klokker M, Mohr T, Kjaer M, Galbo H, Pedersen BK. The natural killer cell response to exercise in spinal cord injured individuals. Eur J Appl Physiol Occup Physiol. 1998;79:106-109. PubMed doi:10.1007/s004210050481

23. Campagnolo DI, Dixon D, Schwartz J, Bartlett JA, Keller SE. Altered innate immunity following spinal cord injury. Spinal Cord. 2008;46:477-481. PubMed doi:10.1038/ sc. 2008.4

24. Nieman DC. Risk of upper respiratory tract infection in athletes: an epidemiologic and immunologic perspective. J Athl Train. 1997;32:344-349. PubMed

25. Moreira A, Delgado L, Moreira P, Haahtela T. Does exercise increase the risk of upper respiratory tract infections? Br Med Bull. 2009;90:111-131. PubMed doi:10.1093/ bmb/ldp010

26. Pyne DB, Gleeson M, McDonald WA, Clancy RL, Perry $\mathrm{C}$, Jr, Fricker PA. Training strategies to maintain immunocompetence in athletes. Int J Sports Med. 2000;21(Suppl 1):S51-S60. PubMed doi:10.1055/s-2000-1452

27. Waterhouse J, Reilly T, Edwards B. The stress of travel. J Sports Sci. 2004;22:946-965, discussion 965-966. PubMed doi:10.1080/02640410400000264

28. Spence L, Brown WJ, Pyne DB, et al. Incidence, etiology, and symptomatology of upper respiratory illness in elite athletes. Med Sci Sports Exerc. 2007;39:577-586. PubMed doi:10.1249/mss.0b013e31802e851a 